\title{
Heart rate variability is associated with emotion recognition: Direct evidence for a relationship between the autonomic nervous system and social cognition
}

\author{
Daniel S. Quintana ${ }^{a, b}$, Adam J. Guastella ${ }^{b}$, Tim Outhred ${ }^{a}$, lan B. Hickie ${ }^{b}$, Andrew H. Kemp ${ }^{a, c}$ \\ a SCAN Research \& Teaching Unit, School of Psychology, University of Sydney, Australia \\ ${ }^{b}$ Brain and Mind Research Institute, University of Sydney, Australia \\ ${ }^{\mathrm{C}}$ CADE Clinic, Discipline of Psychiatry, Sydney Medical School, University of Sydney, Australia
}

\section{Article info}

\section{Full citation:}

Quintana, D. S., et al.,. (2012). Heart rate variability is associated with emotion recognition: Direct evidence for a relationship between the autonomic nervous system and social cognition. IJAP 86(2), 168-172. doi:10.1016/j.ijpsycho.2012.08.012

\section{Keywords:}

Heart rate variability, Emotion recognition, Autonomic nervous system, Social Cognition, Polyvagal Theory, Neurovisceral Integration model.

\section{Correspondence:}

Andrew H. Kemp,, School of Psychology,

The University of Sydney, Australia.

e-mail: andrew.kemp@sydney.edu.au

\section{Abstract}

It is well established that heart rate variability (HRV) plays an important role in social communication. Polyvagal theory suggests that HRV may provide a sensitive marker of one's ability to respond and recognize social cues. The aim of the present study was to directly test this hypothesis. Resting-state HRV was collected and performance on the Reading the Mind in the Eyes Test was assessed in 65 volunteers. HRV was positively associated with performance on this emotion recognition task confirming our hypothesis and these findings were retained after controlling for a variety of confounding variables known to influence HRV - sex, BMI, smoking habits, physical activity levels, depression, anxiety, and stress. Our data suggests that increased HRV may provide a novel marker of one's ability to recognize emotions in humans. Implications for under- standing the biological basis of emotion recognition, and social impairment in humans are discussed.

\section{Introduction}

Impairments in the ability to understand and interpret social information are commonly observed in a variety of psychopathologies including; major depressive disorder (MDD), autism spectrum disorders (ASD), and alcohol use disorders (Baron-Cohen et al., 2000; Demenescu et al., 2010; Hasin et al., 2007). The perception, interpretation and generation of responses to the intention, dispositions and behaviors of others are known as social cognition (Green et al., 2008), and deficits in these processes may underpin social impairment. The recognition of facial expressions and the ability to infer the likely mental states of other people are an important feature of social cognition and this ability may predict functional social capacity beyond more traditional neuro- cognitive assessments that index working memory, psychomotor speed, and attention (Bora et al., 2006). While research on the biological basis of social cognition has highlighted the importance of neural circuitry in the medial frontal cortex (Amodio and Frith, 2006), theories also propose a role for the autonomic nervous system (Appelhans and Luecken, 2006). This study aims to directly test the hypothesis that HRV is related to social cognition.

Porges' polyvagal theory (Porges, 2007) proposes that the mammalian autonomic nervous system (ANS) has evolved to support the survival, reproduction and social engagement of the species. An important feature of this theory is the bidirectional influence of the brain on the body (through the nucleus ambiguus) and the 
body on brain (via afferent feedback to the nucleus tractus solitarius). The most recent development of the human ANS in our phylogenetic history is the myelinated vagus nerve, which facilitates engagement with the environment through its inhibitory influence on the sinoatrial node (i.e., the heart's pacemaker). When an individual is threatened, vagal tone is inhibited, triggering a suite of responses that promotes survival (e.g., increased blood flow to the limbs). By contrast, when the surrounding environment is deemed safe, the "vagal brake" is applied, promoting social behavior and homeostatic functions. The application of the vagal brake slows the heart rate (due to tonic vagal influences on the sinoatrial node), encouraging social interaction. Poor control of the vagal brake may hinder social interaction, as survival behaviors increase sympathetic nervous system's activity. The polyvagal theory proposes that optimal social interaction, which includes the recognition of emotion in faces, is facilitated by a calm physiological state (Porges, 2003). Thus, efficient control of the vagal brake and the ANS allows for rapid engagement and disengagement with others.

ANS function can be indexed non-invasively by heart rate variability (HRV), a measure of beat-to-beat temporal changes in the heart rate. High frequency (HF) HRV represents a rhythmic fluctuation of the heart rate in the respiratory frequency band and has been demonstrated to be an index of parasympathetic control and vagal tone (Berntson et al., 1997). A number of studies have reported reduced HRV in a range of psychiatric illnesses including depression, anxiety, and alcohol use disorders (Kemp et al., 2010, 2012a; Ingjaldsson et al., 2003; Quintana et al., in press-b). As reduced HRV is an index of poor ANS regulation, it may also contribute to the poor social relationships that have been reported in these psychiatric illnesses (Baron-Cohen et al., 2000; Demenescu et al., 2010; Hasin et al., 2007). Prior research has provided some support for this proposal; studies on children with autism have reported poor ANS function, indexed by HRV, relative to those without ASDs (Van Hecke et al., 2009). However, only one study has suggested a relationship between HRV and emotion recognition. In that study, ASD children with low HRV were slower in recognizing emotions in comparison to ASD counterparts with high HRV (Bal et al., 2010). The
Reading the Mind in the Eyes Test (RMET; Baron-Cohen et al., 2001) has been used to index emotion recognition aptitude. This test requires participants to determine what someone is thinking or feeling based on images of the eye region. Poor performance in the RMET has been associated with a number of disorders including autism (Baron-Cohen et al., 2001) and depression (Lee et al., 2005) suggesting that emotion recognition may play a role in the etiology and maintenance of these disorders. The use of this test has also been extended to healthy populations (Domes et al., 2007).

To date, the relationship between emotion recognition and HRV has not been examined in healthy adult participants. The aim of the present study therefore was to examine the relationship between HRV and social cognition, indexed by performance on the RMET, in healthy adult participants taking into consideration other factors that have been demonstrated to influence HRV: sex (Ryan et al., 1994; Sztajzel et al., 2008), BMI (Karason et al., 1999), smoking habits, (Hayano et al., 1990) physical activity (Rossy and Thayer, 1998), depression (Kemp et al., 2010, 2012a), anxiety (Thayer et al., 1996), and stress (Dishman et al., 2000). We hypothesized that HF HRV would be positively associated with emotion recognition accuracy as operationalized by the RMET.

\section{Materials and Methods}

\subsection{Participants}

Sixty-five volunteers ( 35 females, 30 males; mean age \pm $S D, 20.91 \pm 6.16$ ) were recruited from a pool of undergraduate students. Participants received university course credit for their participation and gave written informed consent in accordance with Australian National Health and Medical Research Council guidelines. The University of Sydney Human Research Ethics Committee provided ethical approval for this research (Protocol Number 02-2011/13368). Exclusion criteria included a self-reported history of psychiatric illness or any other serious medical condition (e.g., diabetes; cardiovascular disease). Participants were also excluded if they reported current use of antidepressants. In order to prevent any confounding influences of other substances on psychophysiological functioning, participants were 
asked to abstain from caffeine, cigarettes, alcohol and illicit substances on the day of testing.

\subsection{Instruments}

Participants completed the RMET (Baron-Cohen et al., 2001 ) in order to index emotion recognition aptitude. Participants are presented with 36 images of the eye region of different faces, provided four options for each image (e.g., playful) and are instructed that they should answer each response as quickly as possible. We note that the RMET was originally developed to measure social cognition in adults with autism spectrum disorders (Baron-Cohen et al., 2001) and that prior research has shown that university students perform at ceiling levels on the easy items of this test (Baron-Cohen et al., 2001; Domes et al., 2007). Consequently, we calculated a score weighted for difficulty (RMET diff ) based on performance norms in university students (Baron-Cohen et al., 2001). Items that are more difficult were given more weighting than easy items. For example, $8.7 \%$ of the university students provided an incorrect response for item 9 of the RMET - the easiest item - thus we multiplied item 1 scores by 1.087. In contrast, item 19 (the hardest item) was multiplied by 1.417 to reflect its difficulty as $41.7 \%$ of the university students incorrectly respond to this item (Baron-Cohen et al., 2001). This approach is analogous to previous research that has divided the RMET into easy and difficult items (Domes et al., 2007; Guastella et al., 2010), but takes into account responses on all items.

Additional questionnaires completed by participants included the International Physical Activity Questionnaire (IPAQ; Craig et al., 2003) and the Depression Anxiety and Stress scales (DASS-21; Lovibond and Lovibond, 1995). The IPAQ is a 9-item questionnaire that assesses the energy cost of physical activities by calculating a Metabolic Equivalent Task (MET) score. In this questionnaire participants are asked how much time they devote to physical activities over a range of intensities. In a 12-country validation study, the IPAQ demonstrated acceptable levels of criterion validity (Spearman's $p$ of 0.30 ) and repeatability (Spearman's $p$ of 0.76 ; Craig et al., 2003). The DASS is a 21 -item questionnaire that comprises three subscales indexing de- pression, anxiety and stress, which have demonstrated Cronbach's alphas of .94, .87, and .91 respectively (Antony et al., 1998).

Interbeat intervals (IBI) were measured for $5 \mathrm{~min}$ via the Polar RS800CX (Polar Electro Oy, Kempele, Finland) heart rate monitoring system at $1000 \mathrm{~Hz}$, which wirelessly receives HR data from a chest-strap (twolead) worn by participants. Although there has been some debate regarding the validity of Polar monitors to measure R-R intervals (Quintana et al., in press-a; Wallén et al., 2012), research has demonstrated that Polar monitors can be used interchangeably with ECGs in healthy volunteers (Weippert et al., 2010). In their study, Weippert et al. (2010) recorded R-R intervals using a Polar monitor and an ECG simultaneously. Intra-class correlation coefficients and the Bland-Altman limits of agreement method demonstrated excellent agreement between the Polar monitor and ECG.

\subsection{Procedure}

All participants were tested in the morning (0900 1200 h). Participants completed a questionnaire that measured demographic information, along with the IPAQ and DASS and the RMET. Height and weight were

\section{Table 1. Descriptive statistics for HRV and questionnaires}

\begin{tabular}{|c|c|c|}
\hline Variable & Mean & SD \\
\hline HF HRV & 40.3 & 19.76 \\
\hline RMET & 27.44 & 3.1 \\
\hline RMETDIFF & 33.38 & 3.9 \\
\hline Activity & 3847.95 & 3646.09 \\
\hline Depression & 2.51 & 2.96 \\
\hline Anxiety & 2.14 & 2.93 \\
\hline Stress & 4.94 & 3.72 \\
\hline BMI & 22.31 & 4.37 \\
\hline
\end{tabular}

HF HRV, high frequency heart rate variability; RMET, Reading the Mind in the Eyes Test; RMET diff, Reading the Mind in the Eyes Test weighted for difficulty; Activity, International Physical Activity Questionnaire - Metabolic Equivalent Task; BMI, body mass index. 
also measured to calculate BMI. Following these tasks participant's IBIs were recorded for 5 min while they were relaxed in a seated position after a resting period of 5 min. Participants were breathing spontaneously during the recording period; respiration rate does not affect HRV in resting state recordings (Denver et al., 2007).

\subsection{Data analysis}

Raw data was extracted as a text file and imported into Kubios (version 2.0, 2008, Biosignal Analysis And Medical Imaging Group, University of Kuopio, Finland, MATLAB). Samples were filtered with the low automatic filter and visually inspected for artifacts by a single investigator (DSQ); data quality was high, consistent with resting- state recording conditions. Kubios was then used to calculate HF HRV (0.15-0.4 Hz; normalized units) using the Fast Fourier trans- form. As the total power of the spectral signal is heterogeneous from person to person, it has been recommended that HF data should be presented as normalized values (Pagani et al., 1986). The HF band of frequency domain is influenced almost exclusively by parasympathetic activity and has been argued to be an index of vagal tone (Akselrod et al., 1981; Lane et al., 2009). In addition, HF HRV is largely unaffected by sympathetic blockade by propranolol (Berger et al., 1989) indicating that HF reflects a valid index of parasympathetic activity. To determine whether a relationship between the autonomic nervous system and social cognition was present, HF HRV was entered into a simple regression as a predictor of RMETdiff. Following this, we performed a multiple regression with sex, BMI, smoking habits, physical activity levels, and depression, anxiety, and stress as predictors of HF HRV and saved the standardized residual scores: HF $H R V_{\text {adj. }}$. To estimate effect sizes in the population, confidence intervals for $\mathrm{R}^{2}$ were calculated using the SPSS Noncentrality F Calculator. Finally, in order to determine whether there was a relationship between HF HRV and RMET performance after adjusting for the effects of the covariates, a simple correlation was performed between $H F H R V_{\text {adj }}$ and RMET $T_{\text {diff. Analyses were conducted to }}$ ensure the assumptions of normality, linearity, multicollinearity and homoscedasticity were not violated. According to the Kolmogorov-Smirnov statistic, the HF $\mathrm{HRV}_{\text {adj }}$ variable was normally distributed, thus the analysis conformed to parametric assumptions.

\section{Results}

Descriptive statistics are presented in Table 1 and Pearson bivariate correlation coefficients among measures are presented in Table 2. A linear regression was performed first to assess the ability of HF HRV to predict performance on the RMET. The predictor HF HRV $(B=1.47, B$ SE $=0.611, \beta=0.29, t=2.41,95 \% \mathrm{Cl}$ $[0.25,2.69])$ significantly accounted for $8.4 \%(95 \% \mathrm{Cl}$

Table 2. Pearson bivariate correlation coefficients among measures $(\mathrm{N}=65)$

\begin{tabular}{|c|c|c|c|c|c|c|c|}
\hline & BMI & Sex & Smoking & Activity & Depression & Anxiety & Stress \\
\hline Sex & 0.049 & & & & & \\
\hline Smoking & -0.197 & -0.238 & & & \\
\hline Activity & 0.013 & -0.89 & -0.125 & & & \\
\hline Depression & 0.129 & -0.05 & 0.285 & -0.193 & & \\
\hline Anxiety & 0.137 & 0.07 & 0.007 & -0.18 & $.476^{* \star *}$ & \\
\hline Stress & 0.201 & 0.138 & -0.004 & -0.178 & $.591^{* * *}$ & $.654^{* \star *}$ & \\
\hline HF HRV & 0.102 & $.385^{\star \star}$ & 0.001 & -0.124 & -0.065 & -0.152 & 0.12 \\
\hline RMET & -0.131 & 0.157 & 0.146 & -0.17 & -0.066 & -0.107 & -0.108 \\
\hline
\end{tabular}

${ }^{\star \star *} p<0.001,{ }^{* *} p<0.01,{ }^{*} p<0.05$. 


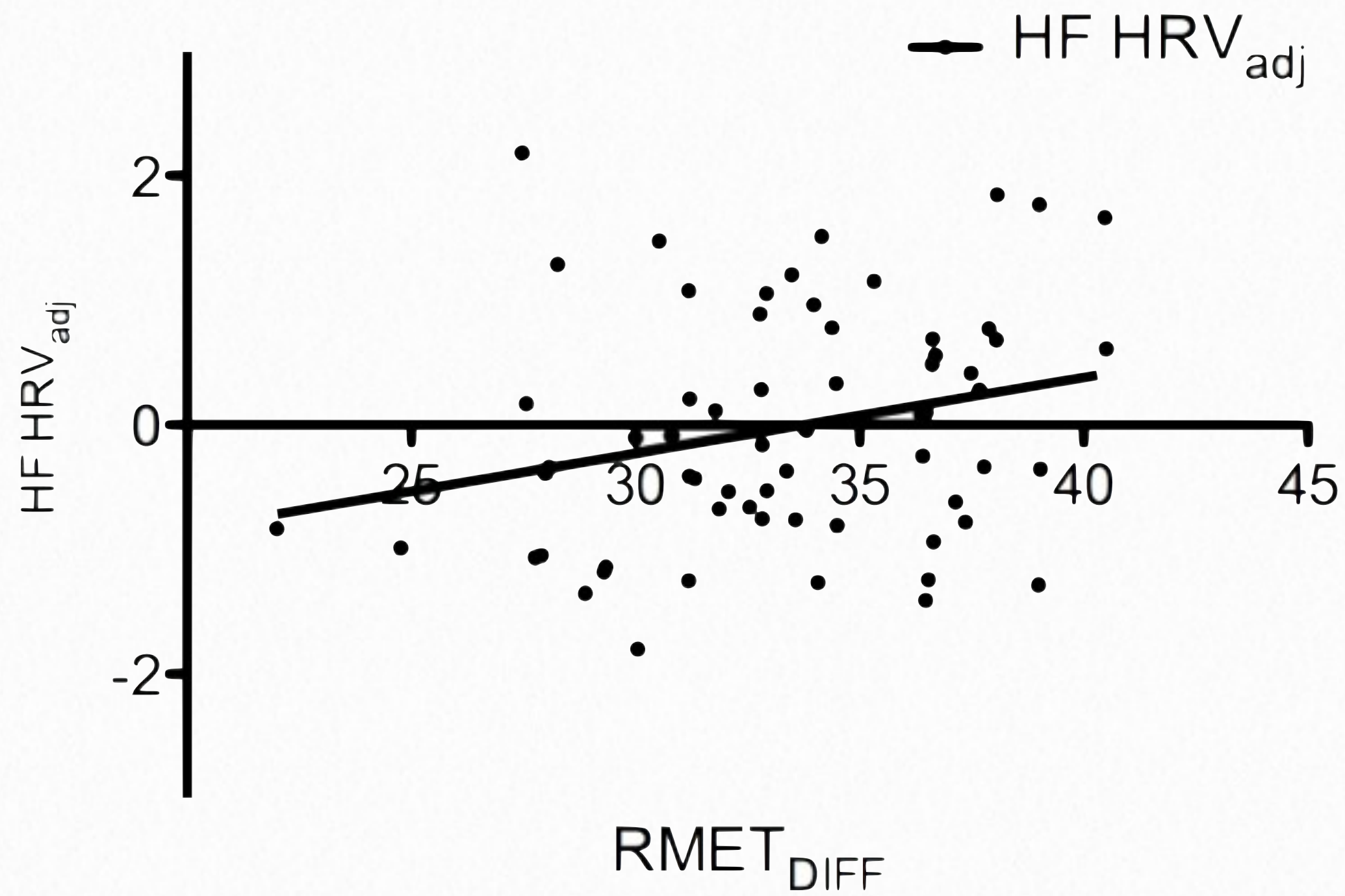

Fig 1. Bivariate relationship between $\mathrm{HF} \mathrm{HRV}_{\text {adj }}$ and RMET $_{\text {diff- }}$

HF HRV ${ }_{\text {adj }}$, high frequency heart rate variability adjusted for covariates; RMET $T_{\text {diff, }}$ Reading the mind in the Eyes test weighted for difficulty. for R2 [> 0\%, 23.0\%]; adjusted $\mathrm{R}^{2}=.07$ ) of the variance on $\mathrm{RMET}_{\text {diff }}$ performance, $F(1,63)=5.793, p=.19$. The variables sex (males coded 0 ; females coded 1$)$, BMI, smoking habits, physical activity levels, and DASS subscores (i.e., DASSDepression, DASS-Anxiety; and DASS-Stress) significantly predicted a total of $26.7 \%\left(95 \% \mathrm{Cl}\right.$ for $\mathrm{R}^{2}$ [2.2\%, 36.5\%]; adjusted $\left.\mathrm{R}^{2}=17.9 \%\right)$ of the variance in $\mathrm{HF}$ HRV, $F(7,58)=3.019, p=.009$. Sex, DASS-Anxiety, and DASS-Stress were significant predictors (Table 3 ). Following this, a correlation coefficient was calculated to assess the relationship between HF adjusted for covariates - HF HRV $\mathrm{Hdj}_{\text {- }}$ and performance on the RMET. There was significant, positive correlation between the two variables, $r=.26, n=65, p<.04$. Therefore, after adjusting HF $\mathrm{HRV}$ for the identified covariates, HF HRV is still associated with performance on the RMET. Fig. 1 displays the spread of scores on HF HRV adj against RMET performance.

\section{Discussion}

This study presents evidence that HRV may provide a novel biological marker of emotion recognition capacity in humans even after controlling for a number of factors known to influence HRV, including physical activity levels, sex, $\mathrm{BMI}$, smoking, and depression, anxiety, and stress. Results indicate that participants who showed higher HRV are more accurate in identifying the emotion ex 
Table 3. Multiple regression analysis for variables predicting HRV

\begin{tabular}{|c|c|c|c|}
\hline Variable & B & B SE & $\beta$ \\
\hline BMI & 0.35 & 0.53 & 0.08 \\
\hline Sex & 14.16 & 4.7 & $.36^{\star *}$ \\
\hline Smoking & 8.45 & 8.66 & 0.12 \\
\hline Activity & $<0.001$ & 0.001 & -0.05 \\
\hline Depression & -0.073 & 1.04 & -0.11 \\
\hline Anxiety & -2.39 & 1.06 & $-.35^{*}$ \\
\hline Stress & 1.85 & 0.9 & $.35^{\star}$ \\
\hline
\end{tabular}

${ }^{* *} p<0.01,{ }^{*} p<0.05$.

pressed on the RMET after adjusting for item difficulty. The results of the present study are consistent with our previous reports of reduced HRV in psychiatric disorders including depression (Kemp et al., 2010), anxiety (Kemp et al., 2012a) and alcohol dependence (Quintana et al., in press-b); all of which are characterized by impairments in social cognition. Given that individuals with these disorders also demonstrate deficits in social cognition the results also provide a greater understanding of the autonomic nervous system's role in the development and maintenance of these disorders and for social cognition in general.

There are a number of theoretical models that provide a foundation on which the findings reported here may be understood. The polyvagal theory highlights the importance of vagal regulation in social behavior, emphasizing the role of the vagus in regulating cardiac output to encourage or discourage social engagement and prosocial behaviors. Our findings indicate that high levels of HRV during a resting state are associated with improved emotion perception. According to the polyvagal theory, through the process of evolution, the brainstem nuclei have become integrated with the muscles of the face and head providing the neurophysiological substrates for social engagement and interaction. Higher HRV reflects not only improved regulatory capacity over autonomic nervous system function but also, a psycho- physiological state compatible with social interaction. Another relevant model to understand the role of ANS regulation in social behavior is the neurovisceral integration model (Thayer and Lane, 2000). While the polyvagal theory focuses on the neural underpinnings of social engagement, highlighting a role for the myelinated vagus, the neurovisceral integration model characterizes a set of neural structures that support behavioral flexibility in responding to a rapidly changing environment. The neurovisceral integration model emphasizes a major inhibitory role for the prefrontal cortex and highlights the relationship between HRV and cognition. Impairment in the prefrontal cortex - a core neurobiological feature of depression - leads to disinhibition of the central nucleus of the amygdala and medullary cardioacceleratory circuits, thus leading to increased heart rate and decreased HRV. Therefore, HRV may be related to social cognition given its relationship with activity in prefrontal neural structures. Together, the polyvagal theory and neurovisceral integration model highlight the important role that is played by the ANS in emotion recognition, and social cognition more generally. Efficient cardiac control facilitates more flexible engagement with the environment and more efficient emotion regulation (Appelhans and Luecken, 2006).

An important component of emotion regulation is being able to determine what the other is thinking by recognizing and interpreting subtle facial cues, which subsequently guide emotional and behavioral responses to others in the environment. A misinterpretation of subtle facial cues may lead to poor quality social interactions commonly described in these disorders, as there is less understanding of the mental states of others. Social impairment may also contribute to disorder maintenance as social support has been found to be a determinant of recovery from mental illness (Hendryx et al., 2008). More generally, our data, together with the extant literature, suggest that social impairment in a variety of psychiatric disorders may be underpinned by reductions in HRV and that otherwise healthy individuals with low HRV may display such impairment. Interestingly, previous work including our own has shown that a hormone and neuropeptide, oxytocin, enhance both HRV (Kemp et al., 2012b; Norman et al., 2011) and RMET performance 
on the difficult items (Domes et al., 2007). It is possible therefore that increases in HRV may underpin the impact of oxytocin on social cognition. The use of oxytocin may also show some promise in the treatment of disorders with deficits in social cognition as oxytocin has been found to increase performance on the RMET in youth with autism spectrum disorders (Guastella et al., 2010).

A limitation of the present study is that no conclusions can be made over the causality - due to study design. That is, it remains unclear whether change in $\mathrm{HF}$ HRV impacts on the capacity to recognize emotion recognition. Future work would benefit from experimental manipulation of HF HRV to determine for example, if reducing HRV compromises emotion recognition. In addition and unexpectedly, we found a significant relationship between the stress subscale of the DASS and HF HRV, such that higher levels of stress predicted increased HF HRV. This finding contrasts with our other finding for anxiety, which indicates that higher levels of anxiety predict decreased HF HRV. Indeed, this latter finding is consistent with the extant literature on psychiatric illness and HRV (Kemp et al., 2010, 2012a; Quintana et al., in press-b). Although this was inconsistent with past research by Dishman et al. (2000) who reported a relationship between perceived emotional stress and HF HRV the present study used a different operationalization of stress (the stress subscale of the DASS), which may index a different facet of stress. Regardless, we note that the population tested in the present study was free from any history of psychiatric illness, that the positive relationship with stress is not clinically relevant and is unlikely to reflect pathological stress.

In summary, this research provides direct evidence to support the relationship between ANS function and emotion recognition, a facet of social communication. This was observed even after controlling for a variety of variables that are known to influence HRV. To our knowledge this is the first study to examine the relationship between HRV and emotion recognition in adults. As reduced HRV has been reported in a number of mental disorders, this result may provide an explanation for the social cognition deficits and highlights the role of the ANS in social cognition and behavior.

\section{Acknowledgment}

The authors DSQ, TO and AHK are supported by an Australian Rotary Health/Hooton family scholarship, an Australian Postgraduate Award (APA), and a NHMRC Career Development Fellowship (571101) respectively. 


\section{References}

Akselrod, S., Gordon, D., Ubel, F.A., Shannon, D.C., Barger, A.C., Cohen, R.J., 1981. Power spectrum analysis of heart rate fluctuation: a quantitative probe of beat-to-bear cardiovascular control. Science 213, 220-222.

Amodio, D.M., Frith, C.D., 2006. Meeting of minds: the medial frontal cortex and social cognition. Nature Reviews Neuroscience 7, 268-277. Antony, M.M., Bieling, P.J., Cox, B.J., Enns, M.W., Swinson, R.P., 1998. Psychometric properties of the 42 -item and 21 -item versions of the Depression Anxiety Stress Scales in clinical groups and a community sample. Psychological Assessment 10, 176.

Appelhans, B.M., Luecken, L.J., 2006. Heart rate variability as an index of regulated emotional responding. Review of General Psychology 10, 229-240.

Bal, E., Harden, E., Lamb, D., Van Hecke, A.V., Denver, J.W., Porges, S.W., 2010. Emotion recognition in children with autism spectrum disorders: relations to eye gaze and autonomic state. Journal of Autism and Developmental Disorders 40, 358-370.

Baron-Cohen, S., Ring, H.A., Bullmore, E.T., Wheelwright, S., Ashwin, C., Williams, S.C.R., 2000. The amygdala theory of autism. Neuroscience and Biobehavioral Reviews 24, 355-364.

Baron-Cohen, S., Wheelwright, S., Hill, J., Raste, Y., Plumb, I., 2001. The "Reading the Mind in the Eyes" test revised version: a study with normal adults, and adults with Asperger syndrome or high-functioning autism. Journal of Child Psychology and Psychiatry 42, 241-251.

Berger, R.D., Saul, J.P., Cohen, R.J., 1989. Transfer function analysis of autonomic regulation. I. Canine atrial rate response. American Journal of Physiology - Heart and Cir- culatory Physiology 256, H142-H152.

Berntson, G.G., Bigger, J.T., Eckberg, D.L., Grossman, P., Kaufmann, P.G., Malik, M., Nagaraja, H.N., Porges, S.W., Saul, J.P., Stone, P.H., van der Molen, M.W., 1997. Heart rate variability: origins, methods, and interpretive caveats. Psychophysiolo- gy 34, 623-648.

Bora, E., Eryavuz, A., Kayahan, B., Sungu, G., Veznedaroglu, B., 2006. Social functioning, theory of mind and neurocognition in outpatients with schizophrenia; mental state decoding may be a better predictor of social functioning than mental state reasoning. Psychiatry Research 145, 95103.

Craig, C.L., Marshall, A.L., Sjöström, M., Bauman, A.E., Booth, M.L., Ainsworth, B.E., Pratt, M., Ekelund, U., Yngve, A., Sallis, J.F., Oja, P., 2003. International physical activity questionnaire: 12-country reliability and validity. Medicine and Science in Sports and Exercise 35, 13811395.

Demenescu, L.R., Kortekaas, R., Boer den, J.A., Aleman, A., 2010. Impaired attribution of emotion to facial expressions in anxiety and major depression. PLoS ONE 5, e15058.

Denver, J.W., Reed, S.F., Porges, S.W., 2007. Methodological issues in the quantification of respiratory sinus arrhythmia. Biological Psychology $74,286-294$.

Dishman, R., Nakamura, Y., Garcia, M., Thompson, R., Dunn, A., Blair, S., 2000. Heart rate variability, trait anxiety, and perceived stress among physically fit men and women. International Journal of Psychophysiology $37,121-133$

Domes, G., Heinrichs, M., Michel, A., Berger, C., Herpertz, S.C., 2007. Oxytocin improves "mind-reading" in humans. Biological Psychiatry 61, 731-733.

Green, M.F., Penn, D.L., Bentall, R., Carpenter, W.T., Gaebel, W., Gur, R.C., Kring, A.M., Park, S., Silverstein, S.M., Heinssen, R., 2008. Social cognition in schizophrenia: an NIMH workshop on definitions, assessment, and research opportunities. Schizophrenia Bulletin: Presented at the Schizophrenia Bulletin, pp. 1211-1220.

Guastella, A.J., Einfeld, S.L., Gray, K.M., Rinehart, N.J., Tonge, B.J., Lambert, T.J., Hickie, I.B., 2010. Intranasal oxytocin improves emotion recognition for youth with autism spectrum disorders. Biological Psychiatry 67, 692-694.

Hasin, D.S., Stinson, F.S., Ogburn, E., Grant, B.F., 2007. Prevalence, correlates, disability, and comorbidity of DSM-IV alcohol abuse and dependence in the United States: results from the National Epidemiologic Survey on Alcohol and Related Conditions. Archives of General Psychiatry 64, 830-842.

Hayano, J., Yamada, M., Sakakibara, Y., Fujinami, T., Yokoyama, K., Watanabe, Y., 1990. Short- and long-term effects of cigarette smoking on heart rate variability. The American Journal of Cardiology 65, 84-88.

Hendryx, M., Green, C.A., Perrin, N.A., 2008. Social support, activities, and recovery from serious mental illness: STARS study findings. The Journal of Behavioral Health Services \& Research 36, 320-329.

Ingjaldsson, J.T., Laberg, J.C., Thayer, J.F., 2003. Reduced heart rate variability in chronic alcohol abuse: relationship with negative mood, chronic thought suppression, and compulsive drinking. Biological Psychiatry, 54, 1427-1436.

Karason, K., Mølgaard, H., Wikstrand, J., Sjöström, L., 1999. Heart rate variability and the effect of weight loss. The American Journal of Cardiology $83,1242-1247$.

Kemp, A.H., Quintana, D.S., Gray, M.A., Felmingham, K.L., Brown, K., Gatt, J.M., 2010. Impact of depression and antidepressant treatment on heart rate variability: a review and meta-analysis. Biological Psychiatry $67,1067-1074$

Kemp, A.H., Quintana, D.S., Felmingham, K.L., Matthews, S., Jelinek, H.F., 2012a. Depression, comorbid anxiety disorders, and heart rate variability in physically healthy, unmedicated patients: implications for cardiovascular risk. PLoS One 7 (2), e30777.

Kemp, A.H., Quintana, D.S., Kuhnert, R.-L., Griffiths, K., Hickie, I.B., Guastella, A.J., 2012b. Oxytocin increases heart rate variability in humans at rest: Implications for social approach-related motivation and capacity for social engagement. PLoS ONE 7 (8), e44014.

Lane, R.D., Mcrae, K., Reinman, E.M., Chen, K., Ahern, G.L., Thayer, J.F., 2009. Neural cor- relates of heart rate variability during emotion. NeuroImage 44, 213-222.

Lee, L., Harkness, K.L., Sabbagh, M.A., Jacobson, J.A., 2005. Mental state decoding abilities in clinical depression. Journal of Affective Disorders $86,247-258$

Lovibond, P.F., Lovibond, S.H., 1995. The structure of negative emotional states: com- parison of the Depression Anxiety Stress Scales (DASS) with the Beck Depression and Anxiety Inventories. Behaviour Research and Therapy 33, 335-343.

Norman, G.J., Cacioppo, J.T., Morris, J.S., Malarkey, W.B., Berntson, G.G., DeVries, A.C., 2011. Oxytocin increases autonomic cardiac control: moderation by loneliness. Biological Psychology 86, 174-180.

Pagani, M., Lombardi, F., Guzzetti, S., Rimoldi, O., Furlan, R., Pizzinelli, P., Sandrone, G., Malfatto, G., Dell'Orto, S., Piccaluga, E., Turiel, M., Baselli, G., Cerutti, S., Malliani,A., 1986. Power spectral analysis of heart rate and arterial pressure variabilities as a marker of sympatho-vagal interaction in man can conscious dog. Circulation Research 59, 178-193. 
Porges, S., 2003. Social engagement and attachment: a phylogenetic perspective. Annals of the New York Academy of Sciences 1008, 31-47.

Porges, S.W., 2007. The polyvagal perspective. Biological Psychology 74, 116-143. Quintana, D.S., Heathers, J. A. J., Kemp, A. H. in press. On the validity of using the polar RS800 heart rate monitor for heart rate variability research. European Journal of Applied Physiology.

Quintana D.S., McGregor, I.S., Guastella, A.J., Malhi, G.S., Kemp A.H. in press. A meta-analysis on the impact of alcohol dependence on shortterm resting state heart rate variability: Implications for cardiovascular risk. Alcoholism, Clinical and Experimental Research.

Rossy, L.A., Thayer, J.F., 1998. Fitness and gender-related differences in heart period variability. Psychosomatic Medicine 60, 773-781.

Ryan, S.M., Goldberger, A.L., Pincus, S.M., Mietus, J., Lipsitz, L.A., 1994. Gender and age- related differences in heart rate dynamics: are women more complex than men? JAC 24, 1700-1707.

Sztajzel, J., Jung, M., Bayes de Luna, A., 2008. Reproducibility and gender-related differences of heart rate variability during all-day activity in young men and women. Annals of Noninvasive Electrocardiology 13, 270-277.
Thayer, J.F., Lane, R.D., 2000. A model of neurovisceral integration in emotion regulation and dysregulation. Journal of Affective Disorders 61, 201-216.

Thayer, J.F., Friedman, B., Borkovec, T., 1996. Autonomic characteristics of generalized anxiety disorder and worry. Biological Psychiatry 39, 255266.

Van Hecke, A.V., Lebow, J., Bal, E., Lamb, D., Harden, E., Kramer, A., Denver, J., Bazhenova, O., Porges, S.W., 2009. Electroencephalogram and heart rate regulation to familiar and unfamiliar people in children with autism spectrum disorders. Child Development 80, 1118-1133.

Wallén, M.B., Hasson, D., Theorell, T., Canlon, B., Osika, W., 2012. Possibilities and limitations of the polar RS800 in measuring heart rate variability at rest. European Journal of Applied Physiology 112, 1153-1165.

Weippert, M., Kumar, M., Kreuzfeld, S., Arndt, D., Rieger, A., Stoll, R., 2010. Comparison of three mobile devices for measuring $R-R$ intervals and heart rate variability: Polar S810i, Suunto t6 and an ambulatory ECG system. European Journal of Applied Physiology 109, 779-786. 